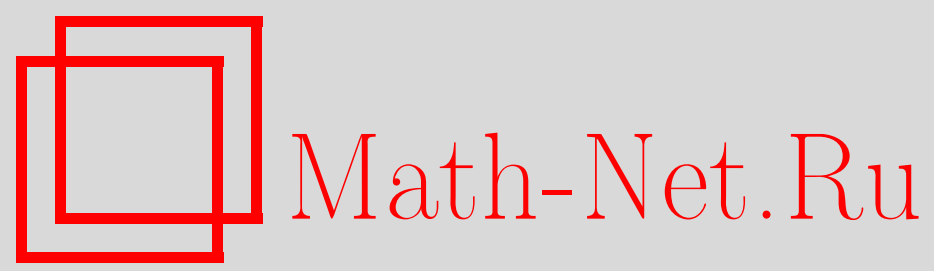

Г. А. Афанасьев, Использование теории массового обслуживания для организации эксплуатации инженерных систем жилых зданий, Вестник ТвГУ. Серия: Прикладная математика, 2019, выпуск 4, 52-64

DOI: https://doi.org/10.26456/vtpmk546

Использование Общероссийского математического портала Math-Net.Ru подразумевает, что вы прочитали и согласны с пользовательским соглашением

http: //www. mathnet.ru/rus/agreement

Параметры загрузки:

IP : 54.162 .127 .20

26 апреля 2023 г., 12:07:50 
УДК 519.218

\section{ИСПОЛЬЗОВАНИЕ ТЕОРИИ МАССОВОГО ОБСЛУЖИВАНИЯ ДЛЯ ОРГАНИЗАЦИИ ЭКСПЛУАТАЦИИ ИНЖЕНЕРНЫХ СИСТЕМ ЖИЛЫХ ЗДАНИЙ}

Афанасьев Г.А.

Московский государственный строительный университет, г. Москва

Поступила в редакцию 21.08.2019, после переработки 23.10.2019.

В последние годы наблюдается повышенный интерес к применению вероятностных методов, в частности, теории массового обслуживания, к оценке эффективности деятельности управляющих компаний при организации эксплуатации технических систем жилых зданий. Основным элементом технической эксплуатации жилых зданий является система планово-предупредительных осмотров и ремонтов. Но даже при их рациональной организации всегда имеется положительная вероятность отказа элементов оборудования, которая зависит не только от факторов старения конструкции. Цель управляющей компании - с одной стороны, не допускать образования слишком большой очереди из экстренных вызовов, а с другой - выполнить все планируемые работы по профилактическому техническому обслуживанию. В статье предложена математическая модель технической эксплуатации жилых зданий, в которой рассматриваются эти две основные функции по обслуживанию жилищного фонда - плановый профилактический осмотр и ремонт технических объектов, а также устранение внезапно возникших неисправностей технического оборудования, в частности аварийных. Считается, что рабочая бригада может приступить к плановому профилактическому ремонту и осмотру, только когда все запросы по внезапно возникшим неисправностям удовлетворены. В качестве основных параметров модели выступают: среднее время между возникновениями неисправностей оборудования, среднее время устранения таких неисправностей, среднее время профилактического осмотра и ремонта одного технического объекта. На основе методов теории массового обслуживания находятся характеристики системы, определяющие качество ее работы, а также границы изменения параметров, при которых система справляется с работой с позиции того или иного критерия.

Ключевые слова: система обслуживания, плановый ремонт, внезапные отказы.

Вестник ТвГУ. Серия: Прикладная математика. 2019. № 4. С. 52-64. https://doi.org/10.26456/vtpmk546 


\section{Введение}

Техническая эксплуатация жилых зданий - это комплекс мероприятий, обеспечивающих наибольшую безотказность всех элементов и систем здания (см., например, [1]- [5]). Основным элементом технической эксплуатации жилых зданий является система планово-предупредительных осмотров и ремонтов. Но даже при их рациональной организации всегда имеется положительная вероятность отказа элементов здания, которая зависит не только от факторов старения конструкции. Отказ может быть вызван случайными обстоятельствами, например недопустимым повышением давления в системах отопления, холодного и горячего водоснабжения и др.

Таким образом, можно выделить две основные функции по техническому обслуживанию жилищного фонда:

- работы по ремонту состояния жилых зданий, профилактическому техническому обслуживанию и ремонтным работам,

- работы по устранению аварийных ситуаций и удовлетворению заявок жильцов на устранение различных неисправностей. Мы будем называть эти работы и соответствующие вызовы экстренными.

Цель управляющей компании - с одной стороны, не допускать образования слишком большой очереди из экстренных вызовов, а с другой, - выполнить все планируемые работы по профилактическому техническому обслуживанию.

Данная статья посвящена, во-первых, выяснению условий на имеющиеся у компании ресурсы, при которых эта цель выполнима, а во-вторых, построению в некотором смысле оптимального поведения.

Наш анализ опирается на теорию массового обслуживания, являющуюся частью теории вероятностей, возникшей из приложений и широко используемой при решении прикладных задач в самых различных областях. Многие исследования как в технологии строительного производства, так и в организации управленческой деятельности основываются на вероятностном подходе, в частности, на результатах теории массового обслуживания (см., например, [1], [6]- [11]). Предлагаемая в данной статья модель не является классической и относится к так называемым системам с вакансиями, изучение которых началось сравнительно недавно (см., например, [12]- [15]). Мы считаем, что работа по плановому осмотру и ремонту начинается только тогда, когда нет экстренных вызовов. Если такая работа началась, то по отношению к экстренным вызовам прибор (рабочая бригада) становится недоступен до момента окончания данного цикла плановьх работ, что и означает вакансию.

\section{1. Постановка проблемы}

Предположим, что управляющая компания (УK) жилищно-коммунального хозяйства $($ KKX) имеет одну или несколько бригад специалистов по обеспечению функционирования технического оборудования (теплоснабжению, водоснабжению, вентиляции и т.д.) жилых зданий. У этих бригад две основные задачи устранение внезапно возникающих поломок оборудования и проведение профилактических осмотров и ремонтных работ с целью обеспечения необходимого уровня 
надежности соответствующих технических систем. Решение указанных задач начинается со сбора и обработки статистических данных, позволяющих получить оценки параметров, определяющих функционирование системы. В качестве этих параметров выступают: $\lambda^{-1}$ - среднее время между последовательными моментами возникновения внезапных поломок оборудования, $\mu^{-1}$ - среднее время ремонта при таких поломках, $\nu^{-1}$ - среднее время профилактического осмотра и ремонта.

Мы предполагаем, что бригада может приступить к такому осмотру, когда нет заявок на экстренный ремонт оборудования. Задача УК выработать такой план профилактических осмотров, при котором, с одной стороны за определенное время $T$ будет осмотрено и восстановлено необходимое число объектов $N$, а с другой, среднее число заявок на ремонт внезапно возникших поломок не превосходит заданный уровень $\delta_{1}$. Как будет показано далее, эта задача может быть невыполнима при некоторых значениях $\lambda, \mu$ и $\nu$. В такой ситуации УК должна принять организационные решения, например, увеличить количество специалистов. Наш анализ будет опираться на методы теории массового обслуживания. Для простоты в этой статье предполагается, что имеется лишь одна рабочая бригада, т.е. один прибор в системе массового обслуживания. Переход к многоканальному случаю не имеет принципиальных препятствий, но технически достаточно сложен. Это будет сделано в следующих работах.

\section{2. Описание математической модели}

Сделаем следующие предположения.

- Интервалы между запросами на экстренный ремонт - независимые экспоненциально распределенные случайные величины $\left\{\tau_{n}\right\}_{n=1}^{\infty}$, т.е. $P\left(\tau_{n} \leq x\right)=1-e^{-\lambda x}$. Это означает, что входящий в систему обслуживания поток требований - пуассоновский (см., например, [16]) и математическое ожидание $M \tau_{n}=\lambda^{-1}$.

- Времена экстренного ремонта поломок $\left\{\eta_{n}\right\}_{n=1}^{\infty}$ - независимые экспоненциально распределенные случайные величины, т.е. $P\left(\eta_{n} \leq X\right)=1-e^{-\mu x}$ и $E \eta_{n}=\mu^{-1}$.

- Имеется одна бригада работников, которая может быть занята либо ремонтом внезапно возникших поломок, либо профилактическим осмотром или ремонтом оборудования. Это означает, что в системе обслуживания один прибор.

- Бригада может приступить к профилактическому осмотру и ремонту, только когда нет запросов на экстренное обслуживание. Мы назовем также запросы требованиями (или клиентами) первого типа. В момент, когда прибор освобождается от требований первого типа, бригада с вероятностью $1-\alpha$ ждет появления следующего экстренного вызова, а с вероятностью $\alpha$ приступает к профилактическому осмотру и (если надо) ремонту какого-нибудь объекта. Считаем, что такие объекты всегда есть в наличии и будем называть их требованиями второго типа. Времена обслуживания таких требований также имеют экспоненциальное распределение с параметром $\nu$ и математическим 
ожиданием $\nu^{-1}$. После окончания обслуживания требования второго типа бригада начинает обслуживать требования первого типа, если они появились в системе, или ждет их появления.

Мы рассмотрим процесс $X(t)=(Q(t), e(t))$, где $Q(t)$ - число требований первого типа в системе в момент $t$, а $e(t)=1$, если в момент $t$ прибор обслуживает требования второго типа (профилактический осмотр и ремонт) и $e(t)=0$, в противном случае. Как известно из теории массового обслуживания (см., например, [16], [17], [18]), при сделанных предположениях $(X(t), e(t))$ является цепью Маркова и на этой основе мы проведем анализ этого процесса и получим операционные характеристики системы.

\section{3. Стационарное распределение процесса $X$}

Для $t \geq 0$ определим вероятности

$$
\begin{gathered}
P_{0 j}(t)=P\{Q(t)=j, e(t)=0\}, \\
P_{1 j}(t)=P\{Q(t)=j, e(t)=1\}, j=0,1,2, \ldots
\end{gathered}
$$

Как известно из теории вероятностей (см., например, [18]), эти функции удовлетворяют системе дифференциальных уравнений. Но нас будет интересовать предельные при $t \rightarrow \infty$ вероятности, т.е.

$$
P_{0 j}=\lim _{t \rightarrow \infty} P_{0 j}(t), \quad P_{1 j}=\lim _{t \rightarrow \infty} P_{1 j}(t),
$$

поскольку именно они определяют операционные характеристики системы на достаточно больших промежутках времени. Эти пределы существуют и задают распределение вероятностей тогда и только тогда, когда $\rho=\frac{\lambda}{\mu}<1$. В этой ситуации $P_{0 j}>0, P_{1 j}>0$ и $\sum_{j=0}^{\infty}\left(P_{0 j}+P_{1 j}\right)=1$ (см., например, [16], [17]).

Последовательности вероятностей $\left\{P_{0 j}, j=0,1, \ldots\right\}$ и $\left\{P_{1 j}, j=0,1, \ldots\right\}$ удовлетворяют системе уравнений баланса [16], которая в нашей модели имеет вид

$$
\begin{aligned}
\lambda P_{00} & =\nu P_{10}+\mu(1-\alpha) P_{01}, \\
(\lambda+\mu) P_{0 j} & =\nu P_{1 j}+\lambda P_{0 j-1}+\mu P_{0 j+1}, \quad j>0,
\end{aligned}
$$

и

$$
\begin{aligned}
& (\lambda+\nu) P_{10}=\alpha \mu P_{01}, \\
& (\lambda+\nu) P_{1 j}=\lambda P_{1 j-1}, \quad j>0 .
\end{aligned}
$$

Для решения этой системы определим производящие функции

$$
\Pi_{0}(z)=\sum_{j=0}^{\infty} z^{j} P_{0 j}, \quad \Pi_{1}(z)=\sum_{j=0}^{\infty} z^{j} P_{1 j}
$$

где переменная $|z| \leq 1$.

Умножая $j$-ое уравнение в (1)и в $(2)$ на $z^{j}$ и складывая по $j$, из (2) получаем

$$
\Pi_{1}(z)=\frac{\alpha \mu P_{01}}{\lambda(1-z)+\nu}
$$


а из (1)

$$
(\lambda+\mu) \Pi_{0}(z)-\mu P_{00}=\nu \Pi_{1}(z)+\lambda z \Pi_{0}(z)+\frac{\mu}{z} \Pi_{0}(z)-\frac{\mu}{z} P_{00}-\alpha \mu P_{01} .
$$

Подставляя (3)в (4), после несложных выкладок получаем

$$
\Pi_{0}(z)=\frac{1}{1-\rho z}\left(P_{00}+\frac{\alpha \beta z}{1+\beta(1-z)} P_{01}\right),
$$

где $\beta=\frac{\lambda}{\nu}$. Для определения $P_{00}$ и $P_{01}$ используем первые уравнения в (1) и (2).

Тогда $P_{01}=\frac{\rho(1+\beta)}{1+(1-\alpha) \beta} P_{00}$ и

$$
\begin{gathered}
\Pi_{0}(z)=\frac{P_{00}}{1-\rho z}\left(1+\frac{\alpha \rho \beta(1+\beta) z}{(1+\beta(1-z))(1+\beta-\alpha \beta)}\right), \\
\Pi_{1}(z)=\frac{\alpha \beta(1+\beta)}{(1+\beta-\alpha \beta)(1+\beta(1-z))} P_{00} .
\end{gathered}
$$

Неизвестная вероятность $P_{00}$ определяется из условия нормировки

$$
\Pi_{0}(1)+\Pi_{1}(1)=1,
$$

откуда

$$
P_{00}=\frac{(1-\rho)(1+\beta(1-\alpha))}{1+\beta+\alpha \beta^{2}}=\frac{1-\rho}{1+\gamma},
$$

где $\gamma=\frac{\alpha \beta(1+\beta)}{1+\beta-\alpha \beta}$.

\section{4. Операционные характеристики системы и их анализ}

Соотношения (5) - (7) позволяют найти важнейшие операционные характеристики, по которым можно судить, насколько УК справляется с решением поставленной проблемы.

Одной из важнейших характеристик системы является среднее число $\bar{q}$ требований первого типа, т.е. среднее число запросов на ремонт вышедшего из строя оборудования. Поскольку

$$
\bar{q}=\sum_{j=0}^{\infty} j\left(P_{0 j}+P_{1 j}\right)=\Pi_{0}^{\prime}(1)+\Pi_{1}^{\prime}(1),
$$

из формул $(5)-(7)$, дифференцируя (5) и (6) и полагая $z=1$, находим

$$
\bar{q}=\frac{\rho}{(1-\rho)}+\frac{\alpha \beta^{2}(1+\beta)}{1+\beta+\alpha \beta^{2}} .
$$

Для требований второго типа заметим, что вероятность завершения обслуживания, т.е. профилактического осмотра и ремонта, за время $\Delta t(\Delta t \rightarrow 0)$ равна $\nu \Pi_{1}(1) \Delta t+o(\Delta t)$, где $\frac{o(\Delta t)}{\Delta t} \rightarrow 0 \quad(\Delta t \rightarrow 0)$. 
Это означает, что среднее число $n(T)$ завершений профилактических осмотров и ремонтов за время $T$ дается выражением

$$
n(T)=\nu \Pi_{1}(1) T=\nu \frac{(1-\rho) \alpha \beta(1+\beta)}{1+\beta+\alpha \beta^{2}} T .
$$

Предположим, что компания желает так организовать работу системы, чтобы среднее число вызовов на срочный ремонт, находящихся в системе, не превышало $\delta_{1}$, а среднее число объектов, профилактический осмотр и ремонт которых завершен за время $T$, был не меньше $N$, т.е.

$$
\bar{q}<\delta_{1}, \quad \nu \Pi_{1}(1)>\frac{N}{T}
$$

Здесь $\delta_{1}$, и $N$ заданы, а в качестве управляющего параметра выступает вероятность $\alpha$. Посмотрим, при каких значениях параметров системы $\lambda, \mu$, $\nu$ этот результат достижим и какие $\alpha$ следует выбирать.

Пусть сначала $\alpha=0$ и посмотрим, когда выполняется первое из условий $(10)$, т.e.

$$
\frac{\rho}{1-\rho}<\delta_{1} \text { или } \frac{\lambda}{\mu}<\frac{\delta_{1}}{1+\delta_{1}} .
$$

Если (11) не выполняется, то компания должна принять организационные решения, приводящие к уменьшению среднего времени устранения внезапно возникших поломок оборудования, т.е. увеличению параметра $\mu$. Это может быть сделано увеличением числа специалистов или повышением уровня их квалификации.

Итак, предположим, что (11) выполняется, т.е.

$$
\mu>\lambda\left(1+\delta_{1}^{-1}\right)
$$

Если компания с вероятностью $\alpha$ направляет бригаду на работы второго типа (профилактический осмотр и ремонт), то в силу (8) первое условие из (10), т.е. $\bar{q}<\delta_{1}$, выполняется, когда

$$
\alpha<\frac{\left(\delta_{1}(1-\rho)-\rho\right)(1+\beta)}{\beta^{2}\left((1+\beta)(1-\rho)+\delta_{1}\right)}=\delta_{2} .
$$

Таким образом, при условии (12) взяв $\alpha=\min \left(1, \delta_{2}\right)$, мы обеспечим выполнение первого неравенства в $(10)$, т.е. $\bar{q}<\delta_{1}$. В силу (9) для выбранного $\alpha$ второе из неравенств в (10) будет выполнено, если

$$
\frac{\nu(1-\rho) \beta(1+\beta) \alpha}{1+\beta+\alpha \beta^{2}}>\frac{N}{T}
$$

или

$$
\frac{\alpha}{1+\beta+\alpha \beta^{2}}>\frac{N}{T \nu} \frac{1}{(1-\rho) \beta(1+\beta)}=a_{1} .
$$

Отсюда

$$
\alpha>a_{1}(1+\beta)+\alpha \beta^{2} a_{1}
$$

Возможны следующие ситуации. 
Если $\beta^{2} a_{1}>1$, т.е. $N>\frac{T \nu(1-\rho)(1+\beta)}{\beta}$, требуемое число профилактических осмотров и ремонтов $N$ не может быть осуществлено при имеющихся параметрах системы $(\lambda, \mu, \nu)$ ни при каких значениях $\alpha$. Следовательно, необходимо принять решения управленческого характера.

Если $\beta^{2} a_{1}<1$, т.е. $N<\frac{T \nu(1-\rho)(1+\beta)}{\beta}$ из (14) следует условие

$$
\alpha>\frac{(1+\beta) a_{1}}{1-\beta^{2} a_{1}}=\delta_{3}=\frac{(1+\beta) N}{T \nu(1-\rho) \beta(1+\beta)-\beta^{2} N},
$$

которое обеспечивает второе из неравенств (10), т.е. требуемое среднее число профилактических ремонтов.

Чтобы выполнялись оба неравенства в (10) в соответствии с (13) и (15), необходимо и достаточно, чтобы

$$
\delta_{3}<\alpha<\delta_{2}
$$

Это возможно, если $\delta_{3}<\delta_{2}$. Используя определения $\delta_{2}$ и $\delta_{3}$, данные соответственно в (13) и (15), получаем неравенство

$$
\frac{N}{T \nu(1-\rho) \beta(1+\beta)-\beta^{2} N}<\frac{\delta_{1}(1-\rho)-\rho}{\beta^{2}\left((1+\beta)(1-\rho)+\delta_{1}\right)},
$$

где $N<\frac{T \nu(1-\rho)(1+\beta)}{\beta}, \delta_{1}<\frac{\rho}{1-\rho}$. Поскольку $\alpha$, будучи вероятностью, лежит в отрезке $[0,1]$, необходимо выполнение неравенства $\delta_{3} \leq 1$, которое, в силу определения $\delta_{3}($ см. $(15))$, имеет вид

$$
N<\frac{T \nu(1-\beta) \beta(1+\beta)}{1+\beta+\beta^{2}}
$$

Очевидно, что из этого неравенства следует, что $\beta^{2} a_{1}<1$.

Таким образом, если выполняются неравенства (17) и (16), выбрав $\alpha \in\left(\delta_{3}, \min \left(1, \delta_{2}\right)\right)$, мы получим систему, удовлетворяющую условиям (10), т.е. среднее число имеющихся в системе запросов на обслуживание (требований первого типа) не превосходит $\delta_{1}$, а среднее число профилактических осмотров за время $T$ не меньше заданного уровня $N$.

Если хотя бы одно из условий (16) или (17) не выполняется, следует предпринять действия управленческого характера - увеличить число специалистов, уменьшить число обслуживаемых объектов, увеличить период $T$ проведения профилактических ремонтов и т.д.

Далее мы приводим план практического применения полученных результатов.

1. На основании реальных наблюдений находятся статистические оценки $\hat{\lambda}, \hat{\mu}, \hat{\nu}$ параметров модели $\lambda, \mu, \nu$. Если окажется, что $\hat{\rho}=\frac{\hat{\lambda}}{\hat{\mu}} \geq 1$, поставленная задача не может быть решена.

2. Выбираются значения $\delta_{1}, T, N$, причем

$$
\begin{gathered}
\delta_{1}>\frac{\hat{\lambda}}{\hat{\mu}-\hat{\lambda}}, \\
N<\hat{\beta}^{-1} T \hat{\nu}(1-\hat{\rho})(1+\hat{\beta}),
\end{gathered}
$$

где $\hat{\rho}=\frac{\hat{\lambda}}{\hat{\mu}}, \hat{\beta}=\frac{\hat{\lambda}}{\hat{\nu}}$. 
3. Вычисляются $\hat{\delta}_{2}$ и $\hat{\delta}_{3}$ соответственно по формулам (13) и (15) с заменой $\rho$ и $\beta$ на их оценки $\hat{\rho}$ и $\hat{\beta}$.

4. Проверяются условия.

Если $\hat{\delta}_{2}>\hat{\delta}_{3}$, то выбирается $\hat{\alpha} \in\left(\hat{\delta}_{2}, \hat{\delta}_{3}\right) \cap(0,1)$. Тогда в системе с параметрами $\hat{\lambda}, \hat{\mu}, \hat{\nu}, \hat{\alpha}$ будет выполнено (10).

Если $\hat{\delta}_{2}<\hat{\delta}_{3}$, выполнение (10) невозможно и нужно принимать решения управленческого характера.

\section{Заключение}

Как и всякая математическая модель, предложенная в статье система массового обслуживания является отображением интересующего нас процесса и используется в целях управления и предсказания. Основная функция этой модели заключается не в том, чтобы описывать явления, а в том, чтобы объяснять их. Модель должна помочь выяснить, каким образом некоторые стороны явления влияют на другие стороны или же на явление в целом. Модель лишь частично отображает действительность. Модель можно считать хорошей, если, несмотря на свою неполноту, она способна предсказывать влияние изменений в системе на общую эффективность всей системы.

Если полученные с помощью модели выводы не удовлетворяют исследователя, она может быть усложнена введением дополнительных связей и параметров.

Заметим, однако, что подобное усложнение не всегда повышает адекватность модели, поскольку требуется статистически оценить большое число новых параметров, что может привести к значительным ошибкам.

Мы рассмотрели простейшую модель, предположив, что имеется всего одна рабочая бригада. Это ограничение может быть снято двумя путями. Если есть $m$ бригад, мы получаем $m$-канальную систему массового обслуживания, анализ которой весьма сложен и формулы для оценки характеристик удается получить лишь при $m=2$. При этом формулы весьма громоздки.

Другой подход состоит в разделении всего множества обслуживаемых объектов на $m$ подмножеств, так что $j$-я бригада занимается техническим обслуживанием объектов из $j$-го множества $j=1,2, \ldots, m$. Тогда мы имеем $m$ одноканальных систем и можем использовать результаты, полученные в данной статье.

Еще одно обобщение нашей модели - изменение политики по отношению к плановым профилактическим осмотрам и ремонтам. Например, предположим, что бригада после освобождения от ремонта внезапно возникших неисправностей ждет случайное время со средним $\zeta^{-1}$. Если за это время запросов на такие ремонты не поступило, бригада начинает плановый осмотр какого-нибудь объекта. Для оценки характеристик такой модели можно воспользоваться формулами (8) и (9), положив $\alpha=\frac{\zeta}{\zeta+\lambda}$.

Другие возможные обобщения будут рассмотрены в следующих работах. 


\section{Список литературы}

[1] Нотенко С.Н., Римшин В.И., Ройтман А.Г. и др. Техническая эксплуатация зданий / под ред. В.И. Римшина, А.М. Стражникова. Учебник. М.: Высшая школа, 2008. 639 с.

[2] Кузин Н.Я., Мищенко В.Н., Мищенко С.А. Управление технической эксплуатацией зданий и сооружений. Учебник. М.: ИНФРА-М, 2017.

[3] Кузин Н.Я. Техническая эксплуатация зданий. Пенза, 1995. 90 с.

[4] Нотенко С.Н. и др. Техническая эксплуатация жилых зданий. М.: Высшая школа, 2000. 497 с.

[5] Рощина С.М., Лукин М.В., Лисятников М.С., Тимахова Н.С. Техническая эксплуатация зданий и сооружений. М., 2018. 232 с.

[6] Байдурин А.Х., Головнев С.Г. Качество и безопасность строительных технологий. Челябинск: Издательство ЮУрГУ, 2006. 433 с.

[7] Чирков В.П. Вероятностные методы расчета мостовых железобетонных конструкций. М.: Транспорт, 1980. 134 с.

[8] Столбов Ю.В. Статистические методы контроля качества строительных работ. М.: Стройиздат, 1982. 87 с.

[9] Спаркис Б.И. Оптимальные расчеты и контрольные значения случайных параметров как средство оптимизации надежности // Проблемы надежности в строительном проектировании. 1972. С. 202-206.

[10] Коуден Д. Статистические методы контроля качества. М.: Физматлит, 1961. $623 \mathrm{c}$.

[11] Dement'eva M. Factors of quality reduction of exploitation of pitched roofs width a cold attic in conditions of dense urban development // MATEC Web Conferences. Vol. 106. 2017. ID 02019. https://doi.org/10.1051/matecconf/201710602019

[12] Sethilinathan B., Kumar J. A study on the behavior of the serever breakdown without interruption in a $M^{[X]}|G(a, b)| 1$ queueing system with multiple vacations and closedown time // Applied Mathematics and Computation. 2012. Vol. 219. Pp. 2618-2633.

[13] Ayyappan G., Karpagam S. An $M^{[X]}|G(a, b)| 1$ queueing system with two heterogeneous service, Server breakdown and repair, Multiple vacation Closedown, Balking and stand-by server // IOSR Journal of Mathematics. 2016. Vol. 12, № 6 . Pp. 56-74.

[14] Selvaraju N., Goswami C. Impatient customers in an $M|M| 1$ queue with single and multiple working vacations // Computers and Industrial Engineering. 2013. Vol. 65, № 2. Pp. 207-215.

[15] Servi L.D., Finn S.G. $M|M| 1$ queues with working vacations $M|M| 1 \mid W V / /$ Performance Evalution. 2002. Vol. 50, № 1. Pp. 41-52. 
[16] Саати Т.Л. Элементы теории массового обслуживания и ее приложения. М.: Издательство "Советское радио", 1971.

[17] Боровков А.А. Вероятностные процессы в теории массового обслуживания. М.: Наука, 1972.

[18] Феллер В. Введение в теорию вероятностей и ее применения. М.: Мир, 1967.

\section{Образец цитирования}

Афанасьев Г.А. Использование теории массового обслуживания для организации эксплуатации инженерных систем жилых зданий // Вестник ТвГУ. Серия: Прикладная математика. 2019. № 4. С. 52-64. https://doi.org/10.26456/vtpmk546

\section{Сведения об авторах}

\section{1. Афанасьев Григорий Александрович}

доцент кафедры жилищно-коммунального комплекса Московского государственного строительного университета.

Россия, 129337, г. Москва, Ярославское шоссе, д. 26. E-mail: gregoria@mail.ru 


\title{
EMPLOYMENT OF QUEUEING THEORY FOR MAINTENANCE OF ENGINEERING SYSTEMS OF RESIDUAL BUILDING
}

\author{
Afanasiev Grigory Aleksandrovich \\ Associate Professor, Moscow State University of Civil Engineering \\ Russia, 129337, Moscow, 26 Yaroslavskoye hw. \\ E-mail: gregoria@mail.ru
}

Received 21.08.2019, revised 23.10.2019.

In resent years has been increasing interest for application of probabilistic methods, in particular queueing theory for estimation of activities of managing companies of residential buildings. Maintenance of residential buildings is a set of measures that ensure the highest reliability of all elements and systems of a building. The main element of the technical operation of residential buildings is a system of scheduled prophylactic inspections and repairs. However even with its rational organization, there is always a positive probability of failure of building elements, which depends not only on the aging factors of the structure. Failure can be caused by accidental circumstances, for example, an unacceptable pressure increase in heating systems, cold and hot water supply systems, etc. The goal of the managing company is, on the one hand, to prevent the formation of a too long queue of emergency calls, and on the other, to complete all planned prophylactic maintenance work. The mathematical model for the technical operation of residential buildings, which considers two main functions for servicing the housing stock - scheduled prophylactic inspection and repair of technical objects, as well as the elimination of sudden malfunctions of technical equipment, in particular emergency ones is proposed. It is considered that the service team can start a scheduled preventive repair and inspection only when all requests for sudden malfunctions are satisfied. The main parameters of the model are: the average time between occurrences of equipment malfunctions, the average elimination time of such malfunctions, and the average time for prophylactic inspection and repair of one technical object. Based on the methods of the queuing theory, the system's characteristics that determine the quality of its work, as well as the boundaries of the change of parameters at which the system copes with the work from the standpoint of a particular criterion are defined.

Keywords: queueing system, prophylactic inspection and repair, sudden failure.

\section{Citation}

Afanasiev G.A., "Employment of queueing theory for maintenance of engineering systems of residual building", Vestnik TvGU. Seriya: Prikladnaya Matematika /Herald of Tver State University. Series: Applied Mathematics], 2019, № 4, 52-64 (in Russian). https://doi.org/10.26456/vtpmk546 


\section{References}

[1] Notenko S.N., Rimshin V.I., Rojtman A.G. et al, Tekhnicheskaya ekspluatatsiya zdanij [Technical exploitation of buildings], Handbook, eds. V.I. Rimshin, A.M. Strazhnikov, Vysshaya Shkola Publ., Moscow, 2008 (in Russian), 639 pp.

[2] Kuzin N.Ya., Mishchenko V.N., Mishchenko S.A., Upravlenie tekhnicheskoj ekspluatatsiej zdanij $i$ sooruzhenij [Management with technical exploitation of buildings and structures], Handbook, INFRA-M Publ., Moscow, 2017 (in Russian).

[3] Kuzin N.Ya., Tekhnicheskaya ekspluatatsiya zdanij [Technical exploitation of buildings], Penza, 1995 (in Russian), 90 pp.

[4] Notenko S.N. et al, Tekhnicheskaya ekspluatatsiya zhilykh zdanij [Technical exploitation of residental buildings], Vysshaya Shkola Publ., Moscow, 2000 (in Russian), $497 \mathrm{pp}$.

[5] Roshchina S.M., Lukin M.V., Lisyatnikov M.S., Timakhova N.S., Tekhnicheskaya ekspluatatsiya zdanij i sooruzhenij [Technical exploitation of buildings and structures], Moscow, 2018 (in Russian), 232 pp.

[6] Bajdurin A.Kh., Golovnev S.G., Kachestvo i bezopasnost stroitelnykh tekhnologij [Quality and safety of ferro-concrete constructions], Izdatelstvo YuUrGU, Chelyabinsk, 2006 (in Russian), 433 pp.

[7] Chirkov V.P., Veroyatnostnye metody rascheta mostovykh zhelezobetonnykh konstruktsij [Probabbilistic methods of calculations of bridge ferro-concrete constructions], Transport Publ., Moscow, 1980 (in Russian), 134 pp.

[8] Stolbov Yu.V., Statisticheskie metody kontrolya kachestva stroitelnykh rabot [Statistical methods of the control of quality of construction works], Strojizdat Publ., Moscow, 1982 (in Russian), 87 pp.

[9] Sparkis B.I., "Optimal calculated and control values of random parametrs as a tool of reliability optimization", Problemy nadezhnosti $v$ stroitelnom proektirovanii [Problem of reliability in building projection], 1972, 202-206 (in Russian).

[10] Kouden D., Statisticheskie metody kontrolya kachestva [Statistical methods of the control of quality], Fizmatlit Publ., Moscow, 1961 (in Russian), 623 pp.

[11] Dement'eva M., "Factors of quality reduction of exploitation of pitched roofs width a cold attic in conditions of dense urban development", MATEC Web Conferences. V.106, 2017, https://doi.org/10.1051/matecconf/201710602019.

[12] Sethilinathan B., Kumar J., "A study on the behavior of the serever breakdown without interruption in a $M^{[X]}|G(a, b)| 1$ queueing system with multiple vacations and closedown time", Applied Mathematics and Computation, 219 (2012), 26182633 .

[13] Ayyappan G., Karpagam S., "An $M^{[X]}|G(a, b)| 1$ queueing system with two heterogeneous service, Server breakdown and repair, Multiple vacation Closedown, Balking and stand-by server", IOSR Journal of Mathematics, 12:6 (2016), 56-74. 
[14] Selvaraju N., Goswami C., "Impatient customers in an $M|M| 1$ queue with single and multiple working vacations", Computers and Industrial Engineering, 65:2 (2013), 207-215.

[15] Servi L.D., Finn S.G., " $M|M| 1$ queues with working vacations $M|M| 1 \mid W V$ ", Performance Evalution, 50:1 (2002), 41-52.

[16] Saaty T.L., Elements of queuing theory with applications, Mc Graw Hill Book Company, New York, 1961, 520 pp.

[17] Borovkov A.A., Stochastic processes in Queuing Theory, Springer-Verlag, New York, 1978, 370 pp.

[18] Feller W., An introduction to probability theory and its applications, John Willy \& Sons Inc., New York, 1966. 